\title{
Displacement Behavior Study of the Shear Stress Effect on the Early Viscous Flow Nature of Fe-B-Nb-Y Metallic Glassy Powder in Spark Plasma Sintering
}

\author{
Sangmin Lee ${ }^{1, *}$, Hidemi Kato ${ }^{2}$, Akihiro Makino $^{2}$ and Akihisa Inoue ${ }^{2}$ \\ ${ }^{1}$ Department of Materials Science, Graduate School of Engineering, Tohoku University, Sendai 980-8577, Japan \\ ${ }^{2}$ Institute for Materials Research, Tohoku University, Sendai 980-8577, Japan
}

\begin{abstract}
$\left(\mathrm{Fe}_{0.72} \mathrm{~B}_{0.24} \mathrm{Nb}_{0.04}\right)_{95.5} \mathrm{Y}_{4.5}$ metallic glassy powders were fabricated with a gas-atomizing method and then densely consolidated by the spark plasma sintering (SPS) technique. Densification behavior during the SPS procedure enables us to understand the thermal behavior and viscous deformation profiles of glassy particles. The glassy powders were consolidated under various temperatures to determine the effect of applied stress on the viscous densification behavior. Consequently, viscous densification commenced under a preset stress of $600 \mathrm{MPa}$ at a flow temperature of $T_{\mathrm{f}}=663 \mathrm{~K}$, which is approximately $248 \mathrm{~K}$ lower than that under a stress of $32 \mathrm{kPa}$, as shown by thermo-mechanical analysis (TMA). Furthermore, even below $T_{\mathrm{g}}$, SEM images demonstrated fine imprintability on the glassy powders during this stress-enhanced viscous densification. [doi:10.2320/matertrans.MBW200851]
\end{abstract}

(Received October 22, 2008; Accepted December 19, 2008; Published February 12, 2009)

Keywords: metallic glass, spark plasma sintering, viscous flow, stress, displacement

\section{Introduction}

Recently, the spark plasma sintering (SPS) technique has attracted considerable attention because of its advantages for consolidating glassy alloy powders without devitrification and for fabricating new glassy alloy composites by blending second crystalline or glassy phases. ${ }^{1-5)}$ Amorphous materials including metallic glass can be crystallized in the vicinity of the crystallization temperature, $T_{x}$, because they are thermodynamically metastable phases. In the supercooled liquid temperature range, $\Delta T_{x}\left(T_{x}-T_{\mathrm{g}}\right)$, glassy materials are focused to achieve thermal stability for both greater glass forming ability (GFA) and viscous workability before the crystallization. It is well known that metallic glasses such as Pd- ${ }^{6-8)}$ and Zr-based glasses ${ }^{9,10)}$ having high thermal stability within $\Delta T_{x}$ could be deformed without devitrification by taking advantage of their viscous flow nature at this temperature range. On the other hand, there have been relatively few reports on Fe-based metallic glasses deformed without crystallization by viscous flow in $\Delta T_{x}$ because of their thermally less stable nature compared with that of the metallic glasses mentioned above. The reason why Fe-based glassy alloys are unstable in the vicinity of $T_{\mathrm{g}}$ has not yet been clarified. Hence, in spite of their other functional merits, ferrous glassy alloys such as $\mathrm{Fe}$ - and Co-based alloys have been mainly studied in thin sheet form for power-related applications such as transformer core materials and stators or rotators for electric motors. ${ }^{11-14)}$

For the purpose of industrial applications of ferrous glassy alloys, sheet-like products, however, should be reformed later to fit the requirements of the devices. Thus, amorphous sheets are rolled up or piled up to make a laminated sheet core, the stator or rotator depending on the usage. Another reason such rolling or piling up is that it reduces the eddy current loss. Thus, ferrous amorphous alloys do not necessarily need to be produced as thick sheets or in bulk form. The effective thickness of the sheet as transformer core material, however, is determined by the frequency of an

*Corresponding author, E-mail: smlee@imr.tohoku.ac.jp alternating current (AC). Considering the frequency of 50 or $60 \mathrm{~Hz}$, skin depth (a measure of the distance an alternating current can penetrate beneath the surface of a conductor) is approximately $0.9 \mathrm{~mm}$ in the case of an iron core. However, the skin depth can be enhanced if the electrical resistivity of the material is increased, by the following relationship:

$$
s \approx \sqrt{2 \rho / \omega \mu}
$$

where $\rho, \omega$, and $\mu$ are electrical resistivity, angular frequency and permeability, respectively. ${ }^{15)}$ Equation (1) clearly indicates that bulk-shaped products can be applied if their electrical resistivity is greatly increased.

On soft magnetic amorphous or glassy alloys, it is so difficult to optimize both GFA and magnetic properties, e.g., saturation magnetization and coercivity, simultaneously. To increase GFA, Inoue's empirical rules, i.e., alloys with over three elements, constituent atoms with size differences of more than $12 \%$, and atoms containing large negative mixing enthalpy are considered. ${ }^{16)}$ However, these rules tend to work negatively for enhancing soft magnetic properties, e.g., degrading high saturation magnetization, high resistivity for lower eddy current loss and so on. Considering the abovementioned facts, separating the processes could be the most effective way to increase GFA and enhance the soft magnetic properties. There might be two ways to separately control those characteristics. One is increasing GFA followed by enhancement of the magnetic properties, and the other is to determine the alloy with the best soft magnetism and then to increase GFA. For GFA, the compositional characteristic is the most dominant factor. Thus, the former seems to be a better solution. However, there are limits to enhancing the magnetic properties without modifying the composition. Thus, if it is possible to overcome a lower GFA using consolidation of glassy powders or ribbons by viscous deformation in the supercooled liquid state without devitrification, the most reasonable alternative would be the latter.

As mentioned above, Fe-based glassy alloys are comparably easier to crystallize during the consolidation process in $\Delta T_{x}$ due to their thermal instability. In this study, we achieved the anomalous reduction of the viscous flow 


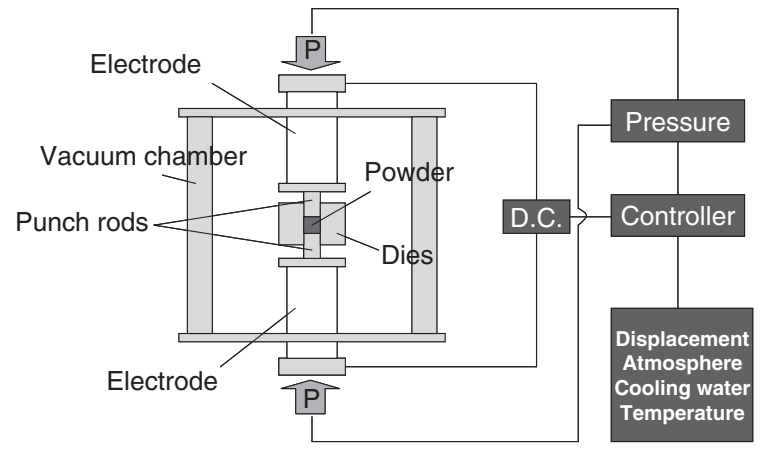

Fig. 1 A schematic of the Spark Plasma Sintering (SPS) method.

temperature by application of pressure on the glassy powders using the SPS method. This enables the fabrication of bulky products without size limitation even for glassy alloys with lower GFA and poor thermal stability. In addition, the electrical resistivity can be controlled by mixing foreign elements or growing surface oxide layers on the powders. Densification behaviors of the glassy powders were investigated to clarify the mechanism responsible for the novel reduction in the flow temperature, $T_{\mathrm{f}}$.

\section{Experimental}

$\left(\mathrm{Fe}_{0.72} \mathrm{~B}_{0.24} \mathrm{Nb}_{0.04}\right)_{95.5} \mathrm{Y}_{4.5}$ glassy powders were fabricated using the gas-atomizing method in an argon atmosphere and consolidated by the SPS technique in a vacuum condition at a heating rate of $0.67 \mathrm{~K} / \mathrm{s}$ up to various temperatures ranging from 673 to $973 \mathrm{~K}$ under a preset pressure of $600 \mathrm{MPa}$ applied with tungsten carbide punches. Figure 1 illustrates a schematic illustration of the SPS method used for consolidating the glassy powders. For the SPS process, displacement profiles including thermal expansion and viscous flow natures were plotted as a function of temperature and compared with the results from thermo-mechanical analysis (TMA) of a cast sample. The cylinder sample cast with dimensions of $\phi 2 \mathrm{~mm} \times 4 \mathrm{~mm}$ was subjected to TMA under a preset stress of $32 \mathrm{kPa}$. The SPSed samples prepared at different temperatures were investigated by an X-ray diffractometer (XRD), a differential scanning calorimeter (DSC) (the results are not shown), and a scanning electronic microscope (SEM) to clarify the effects of the consolidation temperature and stress on the structural state of the powder compacts. More detailed experimental methods for preparing the pre-alloy by the arc-melting and high frequency induction melting methods and for casting samples by the copper mold casting method have been described in a previous paper. ${ }^{17)}$

\section{Results and Discussion}

SPSed $\left(\mathrm{Fe}_{0.72} \mathrm{~B}_{0.24} \mathrm{Nb}_{0.04}\right)_{95.5} \mathrm{Y}_{4.5}$ glassy powder compacts are exhibited in Fig. 2. Each sample was fabricated from approximately $2 \mathrm{~g}$ of the powders at different temperatures. All the samples except for sample A maintained a disk shape with a dimension of $\phi 12 \mathrm{~mm}$ and a thickness of $2-3 \mathrm{~mm}$ depending on the temperature. Sample A which appears to be the powder state without consolidation is however partially densified to maintain its irregular shape, which will later be

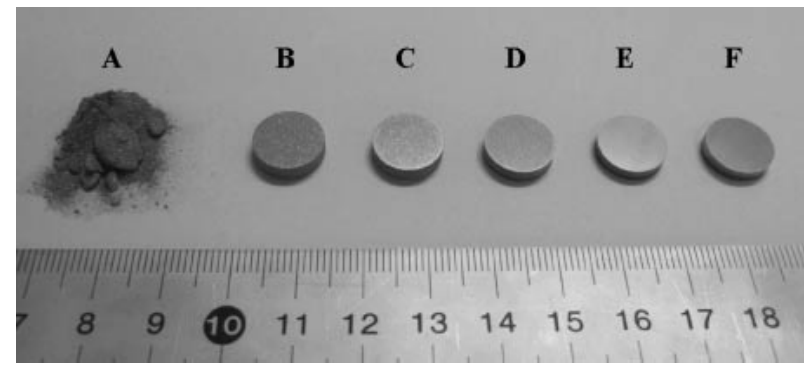

Fig. 2 A photograph of SPSed samples A, B, C, D, E, and F consolidated at $673,723,773,823,873$, and $923 \mathrm{~K}$, respectively.
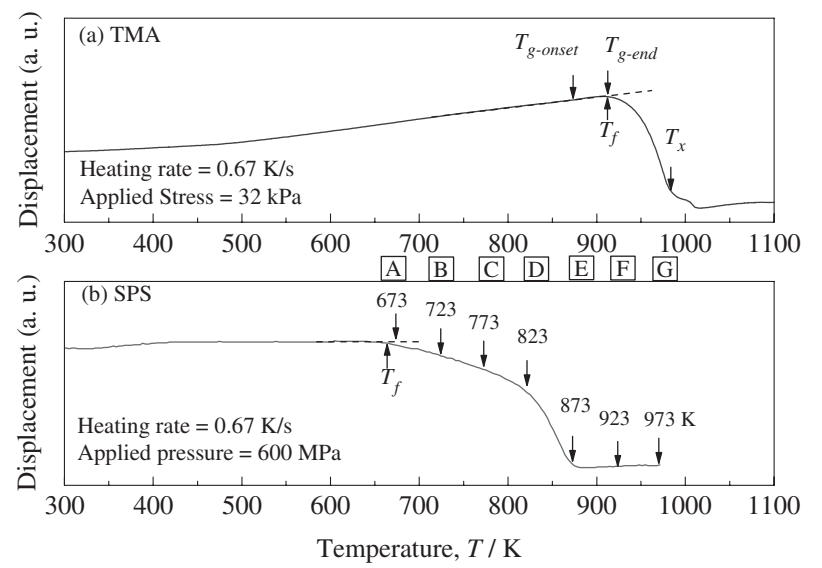

Fig. 3 Displacement curves as a function of temperature obtained by (a) Thermo Mechanical Analysis (TMA) under preset stress of $32 \mathrm{kPa}$ on the cast sample of $\left(\mathrm{Fe}_{0.72} \mathrm{~B}_{0.24} \mathrm{Nb}_{0.04}\right)_{95.5} \mathrm{Y}_{4.5}$ glassy alloy with a dimension of $\phi 2 \mathrm{~mm} \times 4 \mathrm{~mm}$ and (b) Spark Plasma Sintering (SPS) under $600 \mathrm{MPa}$ on the gas-atomized glassy powders packed in $\phi 12 \mathrm{~mm}$ tungsten carbide dies.

shown in detail using SEM images. From sample B to F, thickness was reduced and density gradually increased due to viscous densification.

Figure 3 depicts (a) a change in the probe position of TMA and (b) that in the punch position of SPS at the same heating rate of $0.67 \mathrm{~K} / \mathrm{s}$ under the different stresses of $32 \mathrm{kPa}$ and $600 \mathrm{MPa}$, respectively. It should be noted that the effective stress applied to the glassy powders is considered to be much higher than $600 \mathrm{MPa}$ because the actual area sustaining the applied load should be much smaller than the cross section of the punch due to the spherical shape of powders. The large stress difference between $32 \mathrm{kPa}$ and $600 \mathrm{MPa}$ caused a novel reduction in $T_{\mathrm{f}}$ during SPS. The TMA and SPS results provide important information by comparing the onset and end temperatures of viscous densification with thermal characteristics, $T_{\mathrm{g}}$ and $T_{x}$, determined by DSC. Viscous flow deformation does not occur at the onset point of the glass transition, $T_{\mathrm{g} \text {-onset }}$, but rather at its end point, $T_{\mathrm{g} \text {-end }}$, when the applied pressure is as small as $32 \mathrm{kPa}$, as shown in Fig. 3 (a). On the TMA curve, the probe position increases linearly with increasing temperature due to thermal expansion of the cast sample up to $T_{\mathrm{f}}$, then begins to descend abruptly due to the viscous flow caused by a sudden drop of viscosity. At around $T_{x}$, the viscous deformation is retarded with increasing viscosity caused by the crystallization. On the other hand, as shown in Fig. 3 (b), viscous flow was found to start at $T_{\mathrm{f}}=663 \mathrm{~K}$, which is $210 \mathrm{~K}$ below $T_{\mathrm{g}}$ during SPS under the 
preset pressure of $600 \mathrm{MPa}$. H. J. Jin et al. have pointed out that $T_{\mathrm{g}}$ and $T_{x}$ can shift due to the external pressure, e.g., shear and hydrostatic stresses. ${ }^{18-20)}$ Furthermore, a wider workability due to the reduction in $T_{\mathrm{f}}$ from $T_{\mathrm{g}}$ and the simultaneous enhancement of $T_{x}$ could enable fabrication of bulky products with tiny glassy materials, e.g., powders and ribbons. Kauzmann defined $T_{\mathrm{g}}$ as the temperature at which the time scale of the relaxation is equivalent to that of the experiment. ${ }^{21)}$ Structural relaxation, and thus the glass transition phenomenon, is dominated by the mobility of atoms or defects. The stress effect on atomic jumping probability, $n$, at a given pressure, $P$, in the crystal state is expressed by a Boltzmann function, $n(P)=n_{0} \exp (-P \Delta V / k T)$, where $n_{0}$ is the jumping probability under zero pressure, $k$ is the Boltzmann's constant, $T$ is the temperature, and $\Delta V$ is the activation volume. ${ }^{22)}$ Using this equation, the relaxation time, $\lambda$, can be expressed by the following formula when an external force (e.g. shear stress, $\tau$ ) is applied:

$$
\lambda(P)=\lambda_{0} \exp \left(-\Delta G^{m} \pm \tau \Delta V_{\tau} / k T\right),
$$

where $\Delta G^{m}$ and $\Delta V_{\tau}$ are the activation energy of the jumping motion and the activation volume by shear stress, respectively. The activation barrier would be reduced or enhanced by the direction of $\tau \Delta V_{\tau}$. Jin et al. have drawn the activation volume of $0.126 \mathrm{~nm}^{3}$ for the compression stress of $1.0 \mathrm{GPa}$ on Vit. 4 by substituting the experimental data for this equation. ${ }^{20)}$ This result reveals that collective motions of atoms could be enhanced by external stress. Their assumption and proof by calculation supports our experimental results exhibiting the novel reduction of $T_{\mathrm{f}}$ from the thermally determined $T_{\mathrm{g}}$ of the glassy powders.

To observe the viscous densification state enhanced by large applied stress on glassy powders, samples were consolidated at $673 \mathrm{~K}$ (sample A), $723 \mathrm{~K}$ (B), $773 \mathrm{~K}$ (C), $823 \mathrm{~K}(\mathrm{D}), 873 \mathrm{~K}(\mathrm{E}), 923 \mathrm{~K}(\mathrm{~F})$, and $973 \mathrm{~K}(\mathrm{G})$, followed by XRD and SEM, the results being shown in Figs. 4 and 5, respectively. All the samples except for sample $G$ were glassy even after SPS at each temperature, as shown in Fig. 4. With increasing temperature from A to F, the intensity of the

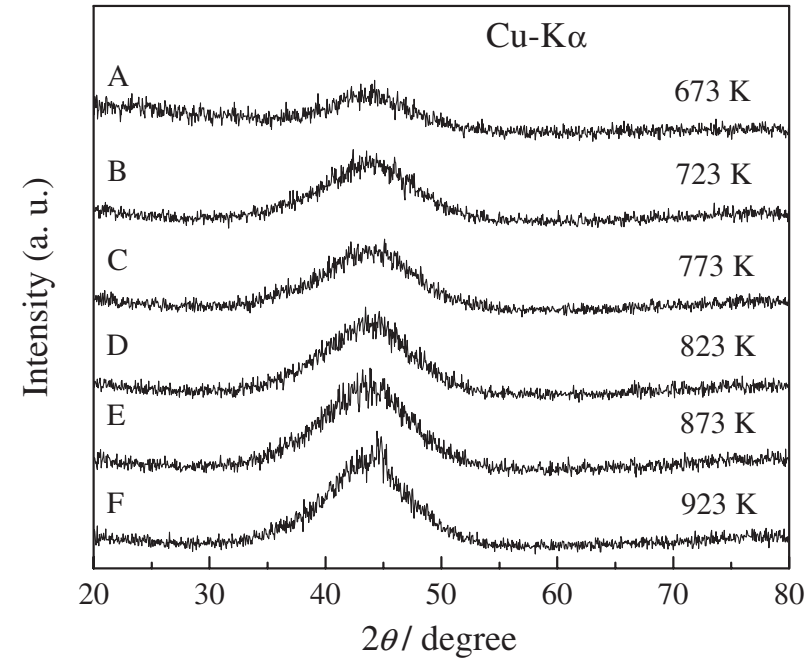

Fig. 4 XRD patterns obtained from samples A, B, C, D, E, F, and G consolidated at $673,723,773,823,873,923$, and $973 \mathrm{~K}$, respectively.

broad peaks became higher at around 35 to 50 degrees, which was caused by the difference in densification of the samples, as seen in Fig. 5. It should be noted that a small peak around 45 degrees corresponds to the surface oxide phase, and thus disappeared when the surface was polished.

As demonstrated by the SEM images, the packing density of the compact increased with increasing temperature from sample A to G. Sample A, which was consolidated at $T_{\mathrm{f}}+10 \mathrm{~K}(=673 \mathrm{~K})$, showed apparent footprints caused by viscous flow on the surface, as seen in Fig. 5, which could be direct evidence of stress-induced viscous flow even below $T_{\mathrm{g}}$. Furthermore, the SEM images of samples B and C well demonstrate the patterned surfaces which were imprinted by the rough surface of the tungsten-carbide punch. From the above results, viscosity of the stress-induced viscous flow is considered to be sufficiently low to enable fine imprinting of the punch surface on the powder surfaces, even at temperatures lower than $T_{\mathrm{g}}$. On the contrary, samples D, E and F consolidated by use of the mirrored surface of the tungsten

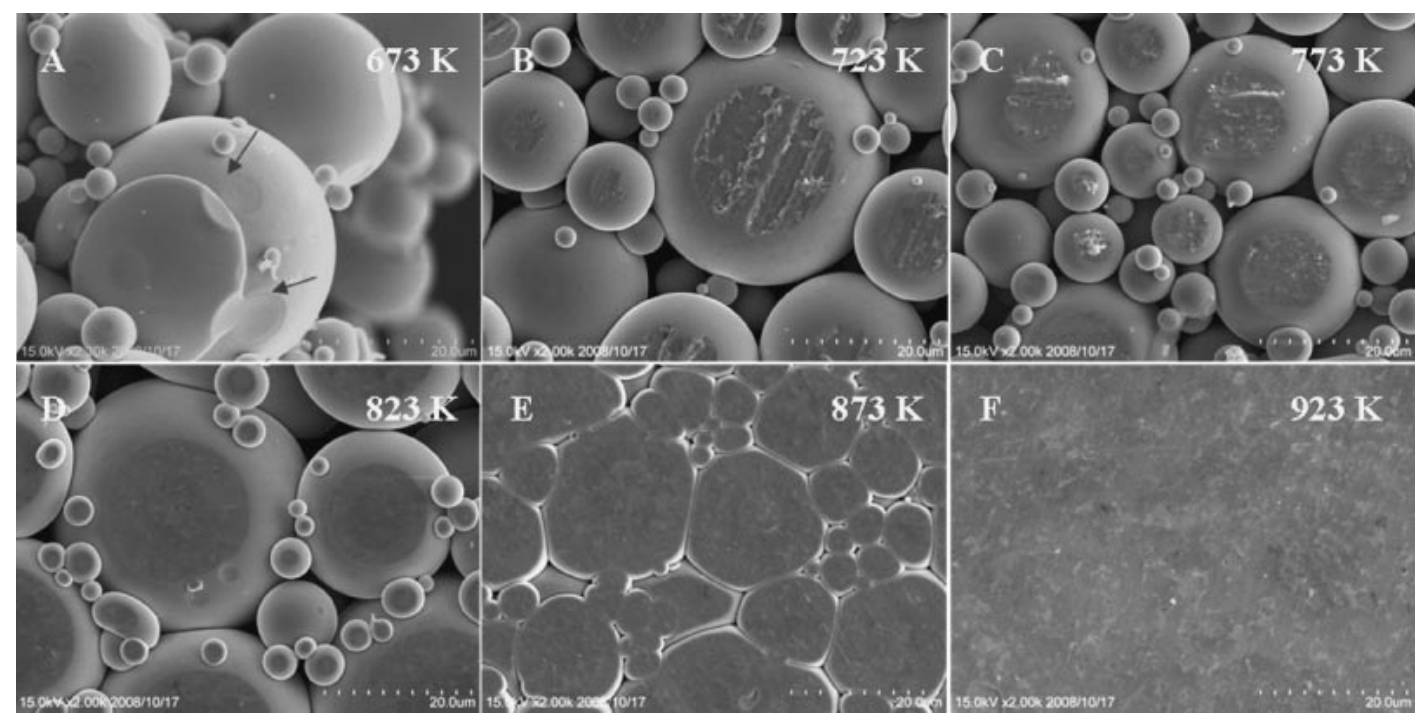

Fig. 5 SEM micro images obtained from the surfaces of SPSed samples A, B, C, D, E, and F consolidated at 673, 723, 773, 823, 873, and $923 \mathrm{~K}$, respectively. 
carbide punch show the mirrored surfaces as a result of the imprinting. These indicate the possibility of the near net viscous shaping of less stable metallic glasses with fine surface-imprinting, even under $T_{\mathrm{g}}$, to maintain the glassy phase.

\section{Summary}

The effect of stress on the viscous densification of $\left(\mathrm{Fe}_{0.72} \mathrm{~B}_{0.24} \mathrm{Nb}_{0.04}\right)_{95.5} \mathrm{Y}_{4.5}$ gas-atomized glassy powders during the SPS procedure was investigated. The results can be summarized as follows.

(1) Viscous densification of the glassy powders was found to start at $T_{\mathrm{f}}=663 \mathrm{~K}$ under a preset stress of $600 \mathrm{MPa}$, which is $248 \mathrm{~K}$ lower than that measured by TMA under $32 \mathrm{kPa}$.

(2) A series of SEM images showed the densification behavior of the glassy powder compacts and exhibited direct evidence for the stress-induced viscous flow in the glassy powder compact consolidated at $673 \mathrm{~K}$, which is $200 \mathrm{~K}$ lower than $T_{\mathrm{g}}(=873 \mathrm{~K})$ determined by DSC.

(3) The patterned or mirrored surfaces imprinted on the compacts demonstrated the fine (micro or nano) imprintability enhanced by the external stress of less stable metallic glasses, even well below $T_{\mathrm{g}}$.

\section{Acknowledgement}

This work was supported by the Global COE Program "Materials Integration (International Center of Education and Research), Tohoku University”, MEXT, Japan.

\section{REFERENCES}

1) D. Roy, S. Kumari, R. Mitra and I. Manna: Intermetallics 15 (2007) 1595.

2) T. Isobe, K. Daimon, T. Sato, T. Matsubara, Y. Hikichi and T. Ota: Ceram. Int. 34 (2008) 213.

3) P. P. Choi, J. S. Kim, O. T. H. Nguyen and Y. S. Kwon: Mater. Lett. 61 (2007) 4591

4) J. K. Kim, H. J. Kim, S. Y. Kim, S. Y. Shin and J. C. Bae: J. Mater. Proc. Tech. 187 (2007) 801.

5) J. K. Lee, H. J. Kim, T. S. Kim, Y. C. Kim and J. C. Bae: J. Alloy. Compd. 434 (2007) 336.

6) H. S. Chen: Acta Metall. 22 (1974) 1505.

7) H. S. Chen: Rep. Prog. Phys. 43 (1980) 353.

8) A. Inoue, N. Nishiyama and T. Masumoto: Mater. Trans. JIM 37 (1996) 181-184.

9) A. Inoue, T. Zhang and T. Masumoto: Mater. Trans. JIM 31 (1990) $177-183$.

10) A. Peker and W. L. Johnson: Appl. Phys. Lett. 63 (1993) 242.

11) A. Makino, T. Kubota, C. Chang, M. Makabe and A. Inoue: Mater. Trans. 48 (2007) 3024-3027.

12) A. Makino, T. Bitoh, A. Inoue and A. L. Inoue: Mater. Sci. Forum 539-543 (2007) 1921.

13) J. Zhang, H. Fujimori, A. Inoue and T. Masumoto: Mater. Sci. Eng. 99 (1988) 35 .

14) A. Inoue and B. L. Shen: Mater. Sci. Eng. A 375-377 (2004) 302.

15) S. Chikazumi: Physics of Ferromagnetism, vol. II (Magnetic Characteristic and Engineering Application, Syokabo, Tokyo, 1984) p. 313.

16) A. Inoue: Mater. Trans. JIM 36 (1995) 866-875.

17) S. Lee, H. Kato, T. Tubota, K. Yubuta, A. Makino and A. Inoue: Mater. Trans. 49 (2008) 506.

18) H. J. Jin, X. J. Gu, F. Zhou and K. Lu: Scr. Mater. 47 (2002) 787.

19) H. J. Jin, X. J. Gu, P. Wen, L. B. Wang and K. Lu: Acta Mater. 51 (2003) 6219.

20) H. J. Jin, J. Wen and K. Lu: Acta Mater. 53 (2005) 3013.

21) W. Kauzmann: Chem. Rev. 43 (1948) 219.

22) M. Paluch, K. L. Ngai and S. J. Hensei-Bielowka: Chem. Phys. 114 (2001) 10872. 